\title{
Polarization-Independent Ultra-Wideband Metamaterial Absorber for Solar Harvesting at Infrared Regime
}

\author{
Asraful Alam ${ }^{1,2, *(\mathbb{C})}$, Sikder Sunbeam Islam ${ }^{1,3}$, Md. Hobaibul Islam ${ }^{1}\left(\mathbb{D}\right.$, Ali F. Almutairi ${ }^{4, *(D)}$ \\ and Mohammad Tariqul Islam $2, *$ (D) \\ 1 Department of Electrical and Electronic Engineering, International Islamic University Chittagong, \\ Chittagong 4318, Bangladesh; sikder_islam@yahoo.co.uk (S.S.I.); hobaib.eee@gmail.com (M.H.I.) \\ 2 Department of Electrical, Electronic \& Systems Engineering, Faculty of Engineering and Built Environment, \\ Universiti Kebangsaan Malaysia, Bangi 43600, Selangor, Malaysia \\ 3 Space Science Centre (ANGKASA), Institute of Climate Change (IPI), Universiti Kebangsaan Malaysia, \\ Bangi 43600, Selangor, Malaysia \\ 4 Electrical Engineering Department, Kuwait University, Kuwait City 13060, Kuwait \\ * Correspondence: asrafulalam.iiuc@gmail.com (A.A.); ali.almut@ku.edu.kw (A.F.A.); \\ tariqul@ukm.edu.my (M.T.I.); Tel.: +60-193-666-192 (M.T.I.)
}

Received: 20 April 2020; Accepted: 1 June 2020; Published: 4 June 2020

\begin{abstract}
This paper presents an ultra-wideband metamaterial absorber for solar harvesting in the infrared regime (220-360 THz) of the solar spectrum. The proposed absorber consists of square-shaped copper patches of different sizes imposed on a GaAs (Gallium arsenide) substrate. The design and simulation of the unit cell are performed with finite integration technique (FIT)-based simulation software. Scattering parameters are retrieved during the simulation process. The constructed design offers absorbance above $90 \%$ within a $37.89 \%$ relative bandwidth and $99.99 \%$ absorption over a vast portion of the investigated frequency range. An equivalent circuit model is presented to endorse the validity of the proposed structure. The calculated result strongly agrees with the simulated result. Symmetrical construction of the proposed unit cell reports an angular insensitivity up to a $35^{\circ}$ oblique incidence. Post-processed simulation data confirm that the design is polarization-insensitive.
\end{abstract}

Keywords: metamaterial absorber; ultra-wideband; polarization independent; solar harvesting

\section{Introduction}

Since the realization of metamaterials, a category of artificially-engineered material designed to exhibit unnatural electromagnetic (EM) phenomena, it has been subjected to rigorous research. Metamaterials (MMs) possess unnatural properties, such as negative permittivity $(\varepsilon)$ and permeability $(\mu)$, an inverse Doppler effect, and so on. Negative values of $\varepsilon$ and $\mu$ permit the energy and phase velocity of a wave to propagate in an inverse direction in the medium, thus resulting in a negative reflective index and forming left-handed material [1]. Leveraging the features of MMs, it has been investigated for different frequency ranges, i.e., $\mathrm{GHz}, \mathrm{THz}$ and optical an frequency regime for most advance applications, such as sensing [2-7], satellite communication [8,9], invisibility cloaking [10,11], super lensing [12,13] and microwave-imaging [14]. Landy et al. [15] first exploited the unique characteristics of MMs, and introduced the first metamaterial perfect absorber (MMPA). MMPAs quickly allured researchers' interest, due to the fact that they can be engineered to absorb a wide range of EM waves, and can be utilized for solar harvesting. Though the solar spectrum of the THz regime is the least-understood portion of the whole EM spectrum [16,17], research on MMPAs includes several studies on solar harvesting in the visible, infrared and ultraviolet regime [18-21]. 
Several polarization-insensitive MMAs have been investigated at different ranges of the $\mathrm{THz}$ spectrum [22-25]. Dhilon and Mittal [26] presented a dually-stacked, gold-stripped resonator that was fabricated on polyamide substrate, which yielded a result of $98 \%$ absorption and a fractional bandwidth of $12.8 \%$ for a narrow frequency range of $0.591-0.672 \mathrm{THz}$. Their illustrated data show that MMPA was insensitive to transverse electric (TE) and transverse magnetic (TM) polarization for the incident angle up to 75 degrees while maintaining absorption above $80 \%$. A similar attempt was demonstrated by Dincer et al. [23] that showed narrowband absorptions of $99.66 \%$ at $268.82 \mathrm{THz}$ in infrared, and $99.9 \%$ at $542.97 \mathrm{THz}$ in visible regimes.

Many researchers have investigated multi-band MMPAs in order to achieve a greater amount of resonant absorption [19,20]. Rufangura and Sabah [18] theoretically designed a dual-band absorber with gold metallic stripes separated from the ground by a dielectric spacer in the visible frequency range. They obtained a peak absorption of $99.9 \%$ at $543 \mathrm{THz}$ and $663 \mathrm{THz}$ for a narrow bandwidth. Liu et al. [27] proposed an ultra-wideband absorber for solar application, but it was extremely sensitive to incident angles. Lee et al. [28] fabricated a flexible multi-band THz MMPA with gold resonator assembled on a Pyrex glass substrate, which yielded two absorption peaks at $0.98 \mathrm{THz}$ and $1.55 \mathrm{THz}$, with respective absorptivities of $93 \%$ and $74 \%$. Tao et al. [29] experimentally characterized a dual-band metamaterial absorber; their simulated design yielded absorption peaks of $85 \%$ at $1.4 \mathrm{THz}$ and $94 \%$ at $3.0 \mathrm{THz}$, although the experimental result of absorption was $70 \%$ at $1.3 \mathrm{THz}$. In both of the above cases, the absorber's bandwidths are low.

However, broadband absorbers are required for many applications, especially for solar harvesting, in terms of efficiency and economy. Several methods may be adopted to extend the bandwidth of absorbers: multiple cells of different structures can be combined into a large cell [30-32] to enhance its absorption range, or multiple cells can be stacked into one [33-35]. Li et al. [36] designed a hybrid structure of a broad-band absorber that provides relative bandwidth up to $82.9 \%$, while Liu et al. [37] presented a tunable broadband absorber at an infrared frequency range. But these methods are constrained by fabrication processes, and dimensional limits, particularly at the $\mathrm{THz}$ regime, where ensuring high precision may lead to higher fabrication costs.

To overcome the limitations of lower bandwidth and lower absorption, this article proposes an ultra-wideband MA (metamaterial absorber), operating at the infrared region for solar energy harvesting. In addition, the study investigates the sensitivity of the unit cell to TE and TM polarization.

\section{Design and Simulation Setup}

The absorption in metamaterial is defined as $A(\omega)=1-\left|S_{11}\right|^{2}-\left|S_{21}\right|^{2}$, where $S_{11}$ and $S_{21}$ are the scattering parameters (S-parameters) for reflection and transmission, respectively. The proposed MA unit cell has three layers, in which a dielectric substrate is sandwiched between two copper layers, as shown in Figure 1a. The metallic copper slabs are patched over the $66.55 \mathrm{~nm}$ thick dielectric GaAs substrate. A continuous metallic plate extrudes the bottom layer of the substrate. The thickness of the continuous plate, $\mathrm{H}$, is maintained at a level higher than the skin depth $(\delta)$ of the material, which is defined as $\delta=\sqrt{2 \rho / 2 \pi f \mu_{R} \mu_{0}}$, in order to ensure zero transmissions. Here, $\rho$ indicates the conductivity of copper, $\mu_{\mathrm{R}}$ indicates the permeability of copper and $\mu_{0}$ expresses the permeability in free space. Hence, the formula for absorption can be condensed into Equation (1).

$$
A(\omega)=1-\left|S_{11}\right|^{2}
$$

An equivalent circuit model is presented to validate the design and to approximate the operating regime of the proposed absorber, as presented in Figure $1 \mathrm{~b}$. The equivalent inductance and capacitance can be estimated using Equations (2) and (3), leveraging techniques from [38-40].

$$
L \approx \mu_{0} D\left(\frac{(w+2 e)}{2 b}+\frac{(2 a+c)-s}{(w+2 e)^{2}+k^{2}}\right)
$$




$$
C \approx \epsilon_{o}\left(\frac{(w+2 e) P^{3}}{10 \pi b^{2}}\right) \ln \frac{a}{f}
$$

where, $\mu_{o}=4 \pi \times 10^{-7} \mathrm{H} / \mathrm{m}$ and $\epsilon_{o}=8.854 \times 10^{-12} \mathrm{~F} / \mathrm{m}$. Equations (2) and (3) yield $1.2424 \times 10^{-7} \mathrm{H}$ inductance and $2.0429 \times 10^{-24} \mathrm{~F}$ capacitance. Consequently, a resonant frequency of $f=1 /(2 \pi \sqrt{ } L C)=$ $315.91 \mathrm{THz}$ is obtained, which is very close to the simulated result presented in the later section.

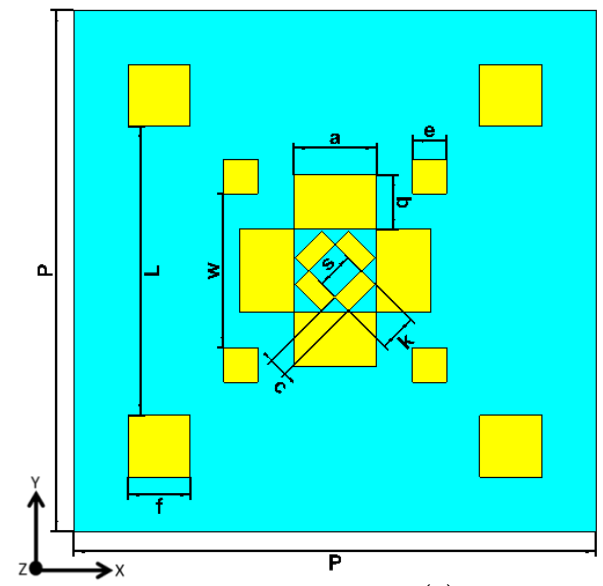

(a)

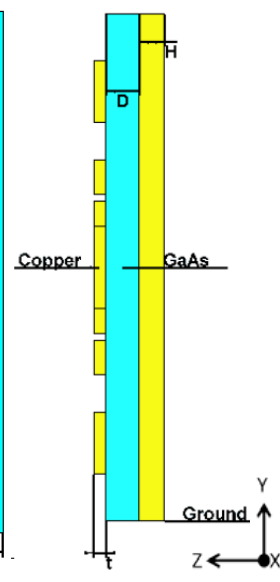

${ }_{4} \zeta$

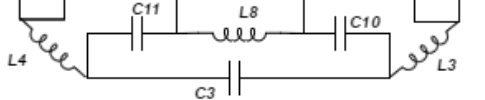

(b)

Figure 1. Geometry of the proposed unit cell: (a) top view and side view, (b) equivalent circuit of the proposed structure.

As a complex design will pose difficulties in fabrication, we have created a minimalistic design that offers horizontal and rotational symmetry.

The proposed design consists of $23.75 \mathrm{~nm}$ thick square- and rectangular-shaped copper patches, as Figure 1a illustrates. Copper is widely used, owing to its excellent electromagnetic properties, while costing little. As for the substrate, the loss tangent and electric permittivity of the GaAs substrate are 0.006 and $\varepsilon=12.94$, respectively, which maintains the thermal conductivity of $401 \mathrm{Wk}^{-1} \mathrm{~m}^{-1}$. The rest of the cellular geometric parameters of the proposed absorber are mentioned in Table 1 . The simulation is performed on a finite integration technique (FIT)-based 'CST Microwave Studio' simulation environment.

Table 1. Structural parameters for proposed unit cell construction.

\begin{tabular}{cc}
\hline Parameter & Value (nm) \\
\hline A & 160 \\
B & 105.6 \\
C & 36.4 \\
K & 72.8 \\
E & 66.4 \\
F & 120 \\
T & 23.7 \\
P & 1010 \\
S & 72 \\
H & 50 \\
D & 66.5 \\
L & 560 \\
W & 298.6 \\
\hline
\end{tabular}

This study chose a perfect electric boundary condition (PEC) along the $X$ plane, a perfect magnetic condition (PMC) along the Y plane, and Open (add space) boundaries along the Z plane. The wave vector $\mathrm{k}$ is perpendicular to the specimen, while the electric and magnetic fields are at a parallel orientation. The frequency domain solver is used during the simulation. 
Although the solar spectrum has an outstretched range of frequencies, this study focuses upon the infrared region (220-360 THz) of the solar spectrum, which covers more than $50 \%$ of the solar spectrum.

\section{Results and Analysis}

The S-parameters are retrieved in a Touchstone $(\mathrm{SnP})$ format from the simulation environment, and are plotted and analyzed in MATLAB.

\subsection{Absorption Characteristics}

The absorption curve can be obtained from Equation (1) However, cross-polarization reflectivity is taken into account during the absorptivity calculation, to ensure that the unit cell is not acting as a polarization converter. The reflection coefficient contains both co-polar and cross-polar components for both TE- and TM-polarized incident waves, as expressed in Equations (4) and (5), respectively.

$$
\begin{gathered}
\left|S_{11}(\omega)\right|^{2}=\left|S_{T E, T E}(\omega)\right|^{2}+\left|S_{T E, T M}(\omega)\right|^{2} \\
\left|S_{11}(\omega)\right|^{2}=\left|S_{T M, T M}(\omega)\right|^{2}+\left|S_{T E, T M}(\omega)\right|^{2}
\end{gathered}
$$

Here, $\left|S_{T E, T E}(\omega)\right|^{2}$ and $\left|S_{T M, T M}(\omega)\right|^{2}$ are the co-polarization reflectivity, and $\left|S_{T E, T M}(\omega)\right|^{2}$ is the cross-polarization reflectivity for TE- and TM-polarized incident wave.

As Figure 2 describes, the cross-polarization reflectivity is insignificant (almost zero in linear magnitude scale) relative to the co-polarization reflectivity, which ensure that the unit cell is not altering the incident wave's polarization throughout the investigated frequency range.

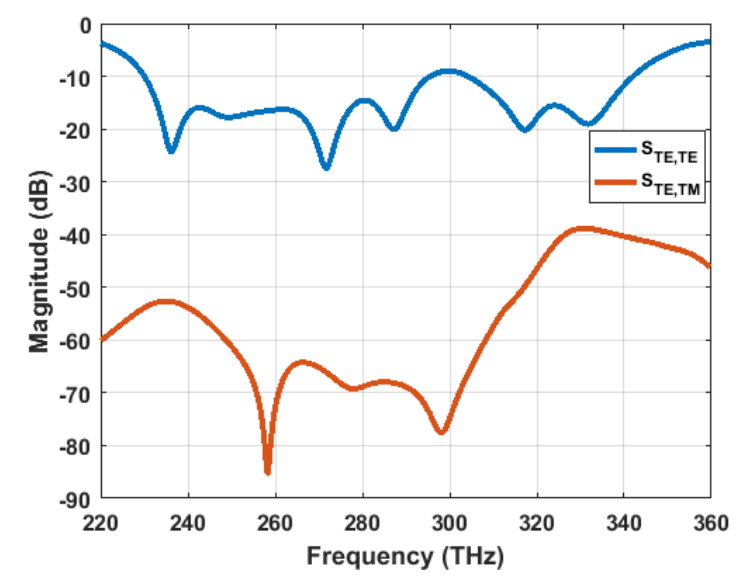

(a)

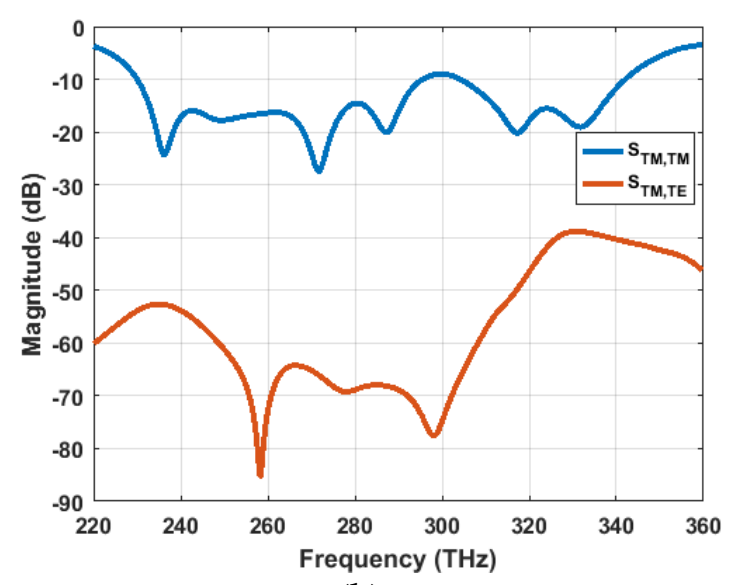

(b)

Figure 2. Co-polarization and cross-polarization components of the reflection coefficient $\left(\mathrm{S}_{11}\right)$ for (a) transverse electric (TE)-polarized incident wave and (b) transverse magnetic (TM)-polarized incident wave.

Moreover, to analyze the absorption characteristics of the proposed MA, we have used the relative absorption bandwidth (RAB), defined as

$$
B_{r}=\frac{2\left(f_{u}-f_{l}\right)}{\left(f_{u}+f_{l}\right)}
$$

Here, $f_{u}$ and $f_{1}$ are the upper and lower limits of the frequency range, within which the absorption is above $90 \%$. From Equation (6), the proposed design offers absorption over $90 \%$, maintaining a $25.602 \%$ relative bandwidth in the frequency range of $229.9-297.4 \mathrm{THz}$. It offers a $12.289 \%$ relative bandwidth, within $303.2-342.9 \mathrm{THz}$, as Figure 3 portrays. 


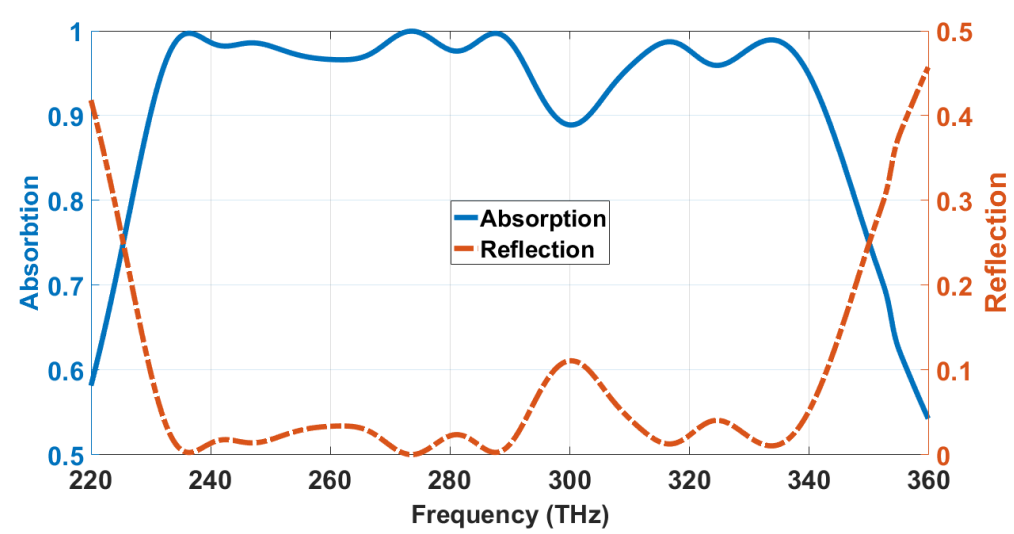

Figure 3. Absorption and reflection spectra of the proposed structure.

Therefore, the proposed MA offers total relative bandwidth, $\mathrm{BRAB}=37.89 \%$, with above $90 \%$ absorption, characterizing it as an ultra-wide band (UWB) absorber.

\subsection{Polarization Independence and Angular Stabiltiy}

It is highly desirable for an ultra-wideband absorber to be polarization-independent and insensitive to any incident angle for many solar harvesting applications. We have investigated the proposed structure under the TE-, TM- and transverse electromagnetic mode (TEM)-polarized wave. The unit cell yields identical responses, regardless of the polarization mode, due to the horizontal and rotational symmetry of the proposed MA structure, as depicted in Figure 4.

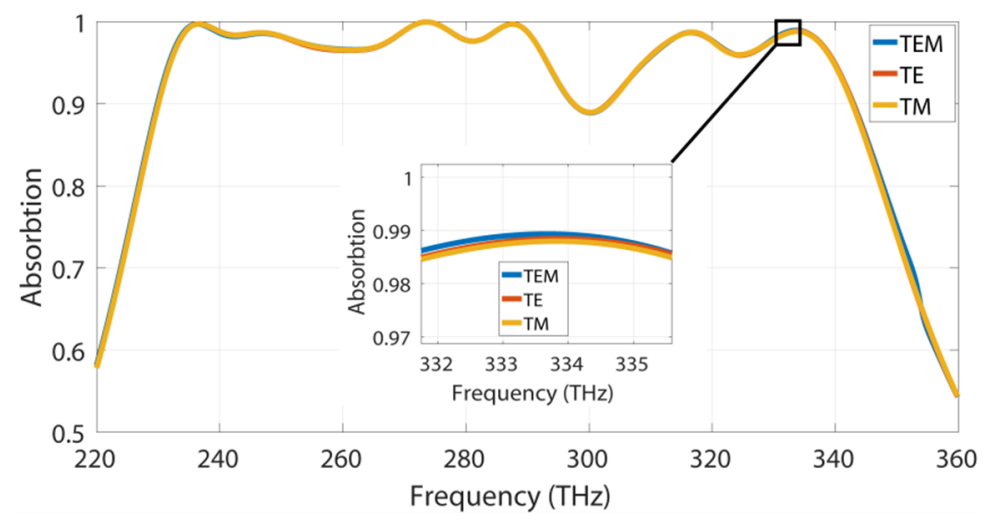

Figure 4. Absorption characteristics under the transverse electromagnetic mode (TEM)-, TE- and TM-polarized wave.

Varying the polarization angle $(\varphi)$ from $0^{\circ}$ to $90^{\circ}$, with a step size of $22.5^{\circ}$, is deployed to investigate polarization angle sensitivity. The unit cell shows excellent polarization angle insensitivity for the aforementioned polarized waves, as Figure 5 illustrates.

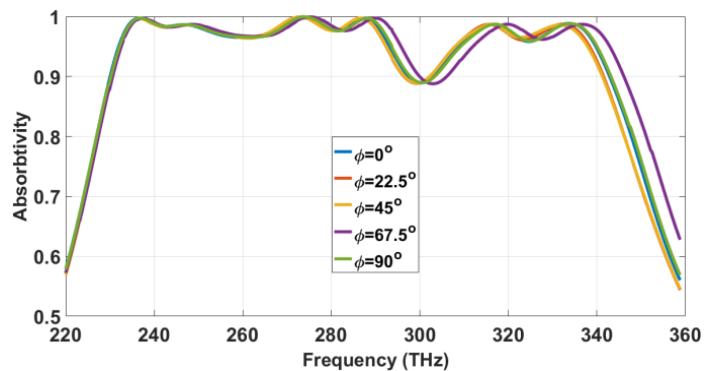

(a)

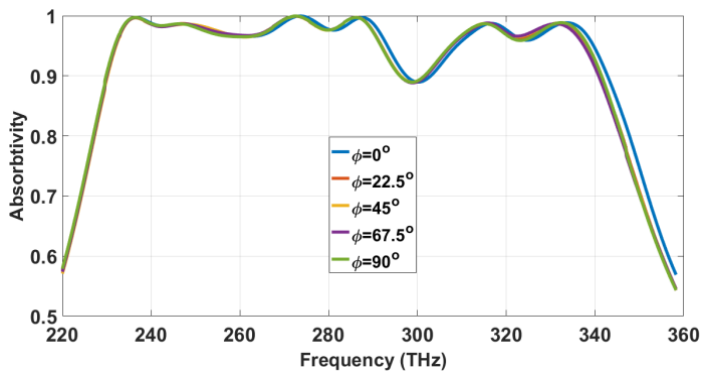

(b)

Figure 5. Polarization angle sensitivity at (a) the TE mode and (b) TM mode. 
Furthermore, angular stability is investigated for the varying incident angle $\theta$ at $\varphi=0$ degrees. At the TE mode, absorptivity degrades as the incident angle approaches $90^{\circ}$. Thi occurs because the decrement of the horizontal component of the E-field with the increment of the incident angle effectively reduces electrical resonance, which leads to a gradual degradation in absorptivity. However, the MA maintains high absorptivity up to $50^{\circ}$ for a wide portion of the frequency range, and it shows excellent angular insensitivity up to $35^{\circ}$, as Figure 6 a demonstrates.

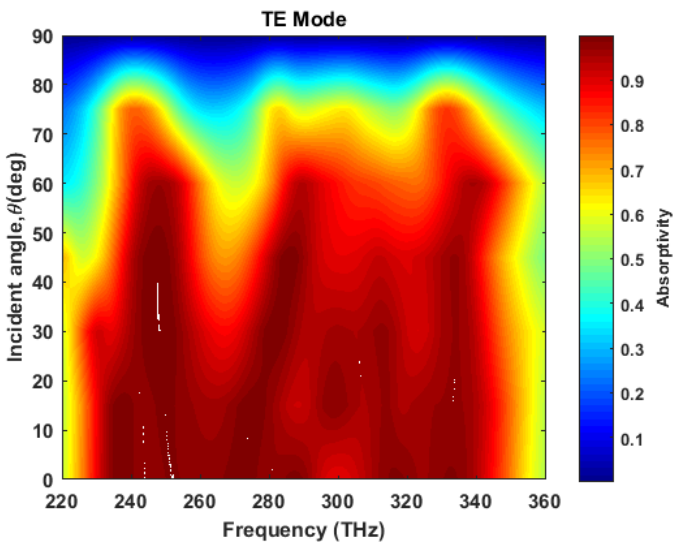

(a)

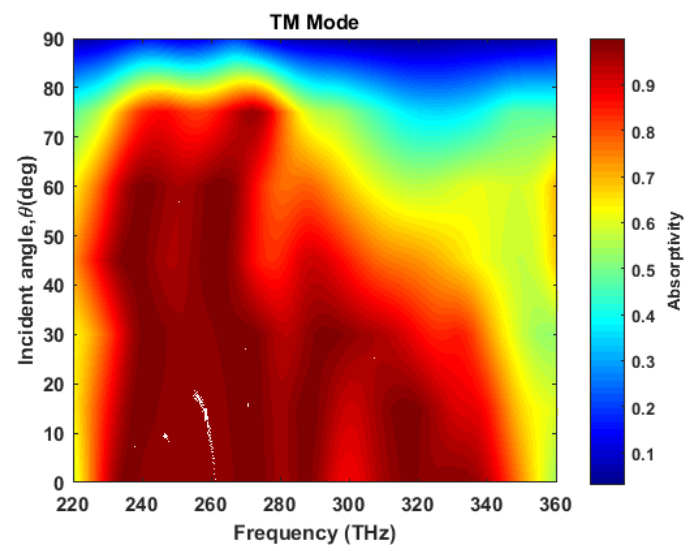

(b)

Figure 6. Angular sensitivity to varying incident angle, $\theta$ at (a) TE mode and (b) TM mode.

A similar degrading phenomenon is observed with the increment of incident angle and frequency at the TM mode (Figure 6b). However, at lower frequencies, the structure shows insensitivity for incident angles as large as $75^{\circ}$. Overall, the MA exhibits insensitivity to an incident angle up to $35^{\circ}$ for the TE and TM mode.

\subsection{Dependence on Geometric Parmeters}

This section investigates the effect of cellular parameters on the absorption characteristics of the proposed absorber. First, the thickness of GaAs substrate (D) was examined. As seen in Figure 7a, the absorption curve introduces a left shift toward lower-frequency spectra, as the thickness of the substrate increases from $63.555 \mathrm{~nm}$ to $75.55 \mathrm{~nm}$ with a step size of 3 . This phenomenon occurs due to the change in the equivalent capacitance between the metallic slabs and the ground plane. The resonator layer of the proposed structure induces a series capacitance between copper patches.

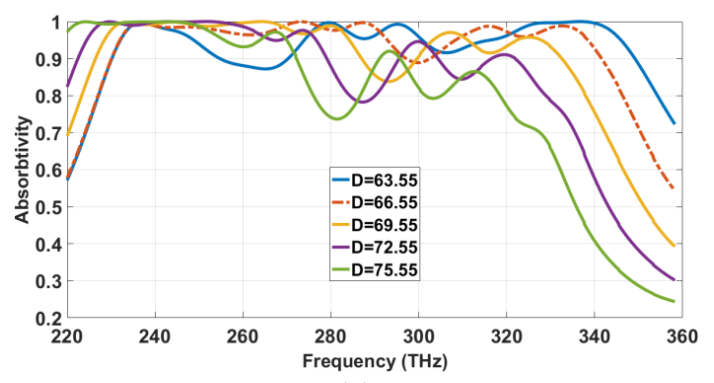

(a)

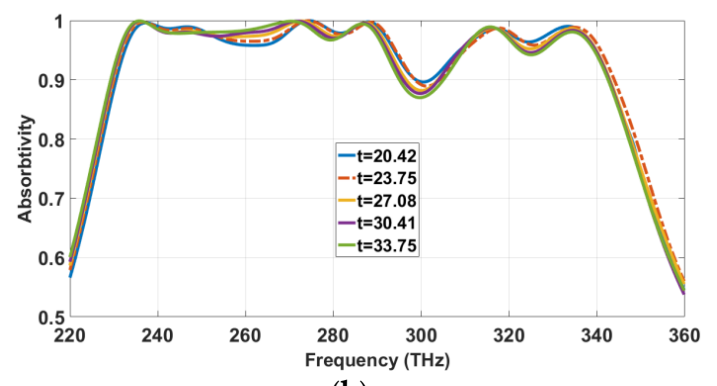

(b)

Figure 7. Absorption characteristics of proposed MA for different thicknesses of (a) dielectric substrate (D) and (b) metallic copper resonator (t).

As thickness D increases, the capacitance decreases for each copper slab. Consequently, the equivalent series capacitance increases for the whole metallic layer, resulting in a lower resonant frequency, which explains the left shift of absorptivity toward lower-frequency spectra.

Meanwhile, the distance between the two metallic layers also increases, which effectively reduces magnetic resonance [41], hence induces a steep fall in absorptivity. 
Consequently, the thickness of the copper films ( $\mathrm{t}$ ) is altered from $22.420 \mathrm{~nm}$ to $33.750 \mathrm{~nm}$ with a step size of $3.3 \mathrm{~nm}$, as Figure $7 \mathrm{~b}$ shows. Similar to the thickness response of dielectric, the absorptivity tends to shift left (toward lower-frequencies) as the thickness (t) increases. Since the equivalent capacitance increases with thickness, its absorptivity therefore moves toward lower-frequency spectra. The MA yields an optimized absorption for $\mathrm{t}=23.75 \mathrm{~nm}$, as the dotted line indicates.

Subsequently, a parameter sweep was conducted on the lateral distances between the copper patches (denoted by parameters W and L in Figure 1a), in order to investigate the effect of gap capacitance between the metallic copper plates.

The absorption characteristics remain almost identical over the whole sweep, from $W=280$ to $\mathrm{W}=303 \mathrm{~nm}$ with a step size of $5 \mathrm{~nm}$, as depicted in Figure 8. However, a trend of decreasing bandwidth is observed as the distance $\mathrm{W}$ increases. The yellow-dotted absorption curve for $\mathrm{W}=293.60$ shows an exceptionally higher relative absorption bandwidth than the others, while maintaining a decent absorption rate over the frequency spectrum.

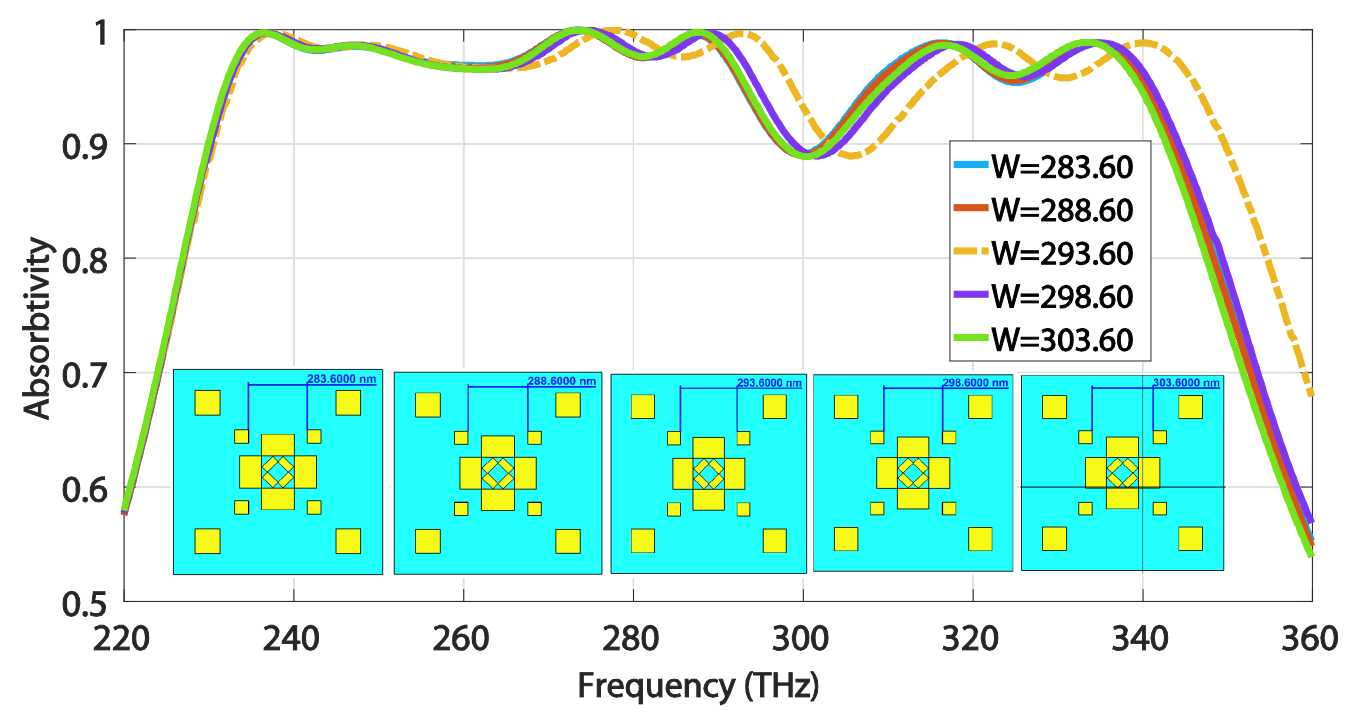

Figure 8. Absorption sensitivity to the lateral distance denoted by W.

As for lateral distance $\mathrm{L}$, the absorption rate noticeably declines with the increment of $\mathrm{L}$ with a step size of $10 \mathrm{~nm}$, as depicted in Figure 9. The absorption curve tends to shift left (toward lower-frequency regions) as the capacitance between the gap drops with distance.

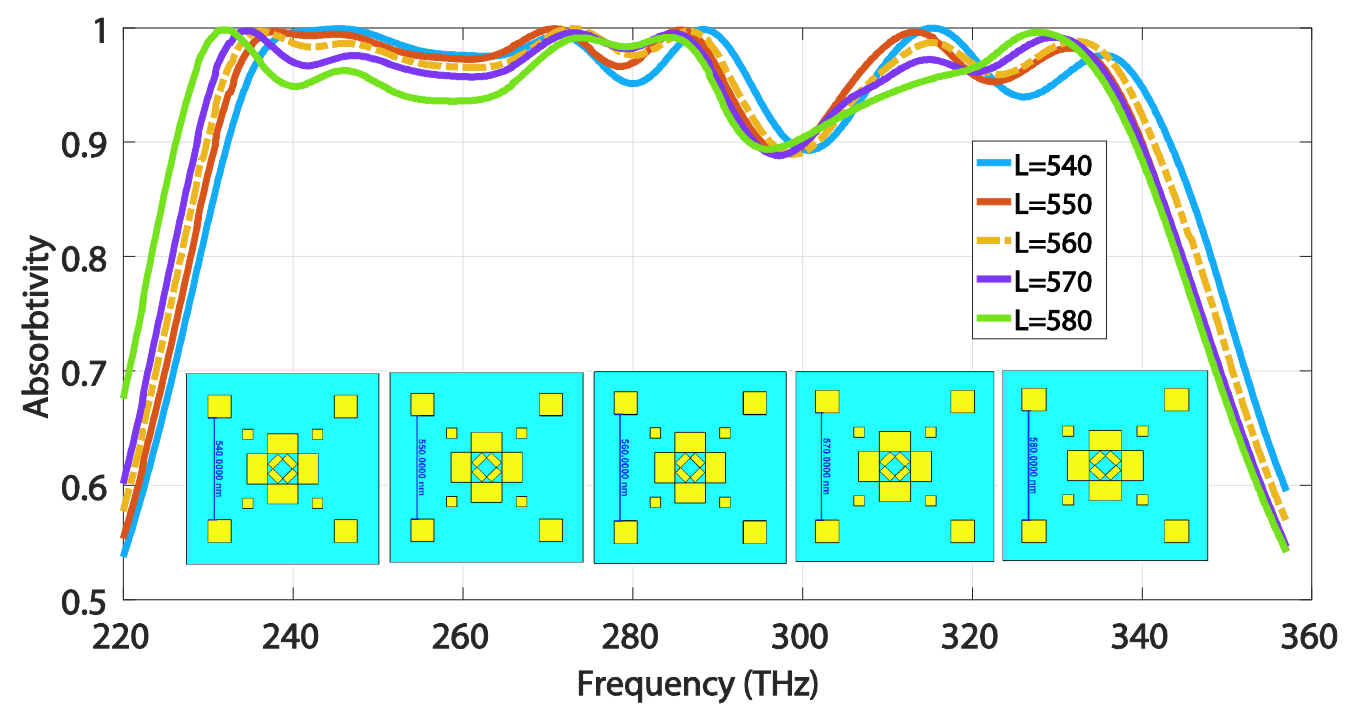

Figure 9. Absorption sensitivity to the lateral distance denoted by L. 
The relative absorption bandwidth at over $90 \%$ absorption remains almost identical throughout the sweep, yet absorption above $95 \%$ dramatically drops with the escalation of distance. The optimized absorption curve is indicated by the dotted line for $\mathrm{L}=560 \mathrm{~nm}$. The structure notably exhibits a similar frequency shifting trend for both varying lateral distances, $\mathrm{W}$ and $\mathrm{L}$. The reason behind this phenomenon is that, as the distance increases, the inter-metallic gap capacitance of each slab decreases; consequently, the equivalent series capacitance of the whole metallic layer intensifies, thereby introducing a shift toward lower resonant frequency spectra, and vice-versa.

\subsection{Mechanism of Absorption}

To realize the nature of the absorption behavior, the surface current and the electric field (E-field) are analyzed at two resonant frequencies $273.76 \mathrm{THz}$ and $333.72 \mathrm{THz}$, with peak absorption. Perfect adsorption was achieved at resonance frequencies due to the simultaneous occurrence of electric resonance and magnetic resonance-i.e., when the electric and magnetic responses of the absorber match with that of the incident wave.

It can be observed from Figure 10 that the MA induces a high concentration of circulating current at the edge of the copper resonators. A closer examination reveals the existence of parallel and anti-parallel surface currents moving around the copper slabs, which introduces electric resonance to the absorber. Meanwhile, the circulating current produces magnetic resonance [18].

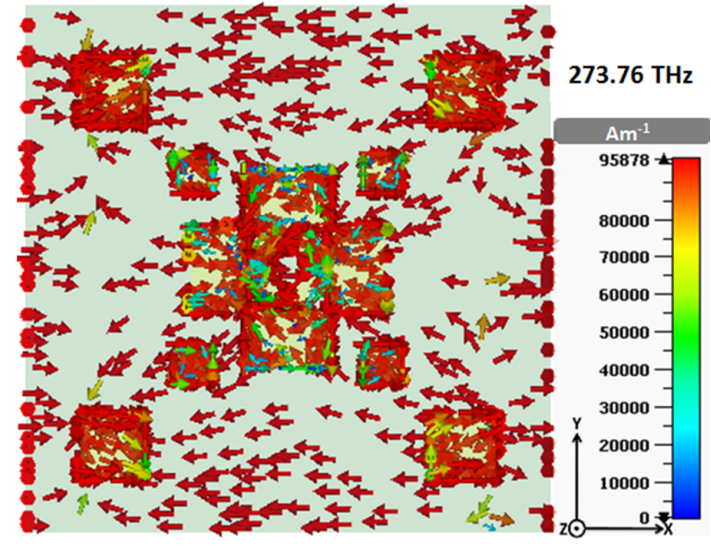

(a)

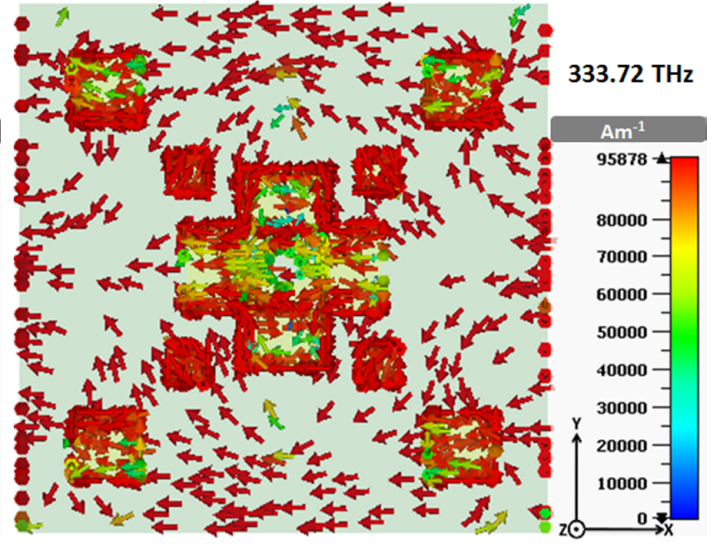

(b)

Figure 10. Surface current distribution of the metallic layer at (a) $273.76 \mathrm{THz}$ and (b) $333.72 \mathrm{THz}$.

Another approach to realizing the absorption process is to analyze the E-field distribution at resonant frequencies. In Figure 11, it seems that the E-field is denser at the edges of copper slabs, such that it acts like electric dipoles, which are accountable for electrical resonance. Additionally, the strong dipole-like E-field creates surface charges that oscillate with the electric field, thus resulting in magnetic resonance [18].

Furthermore, power loss distributions at similar resonant frequencies were investigated in order to understand the trend of absorption in dielectric and the metallic layer. It can be concluded from Figure 12 that the copper resonators are mostly accountable for the wideband absorption, even though GaAs has a low loss tangent (0.0006). The reason behind this is, the metallic layer tends to be lossier than the dielectric at the infrared frequency regime [41], which holds the metallic layer more responsible for wideband absorption. Synthesis of the proposed structure can be performed through an extensively used photolithography technique. However, photolithography requires a complicated procedure, including photo-masking of the samples. Murata et al. [42] introduced a super-fine ink-jet (SIJ) printer, which is capable of fabricating three-dimensional nano-structures with an accuracy analogous to photolithography. This method may be adopted to avoid copper-oxide formation during the synthesis of the structure. 


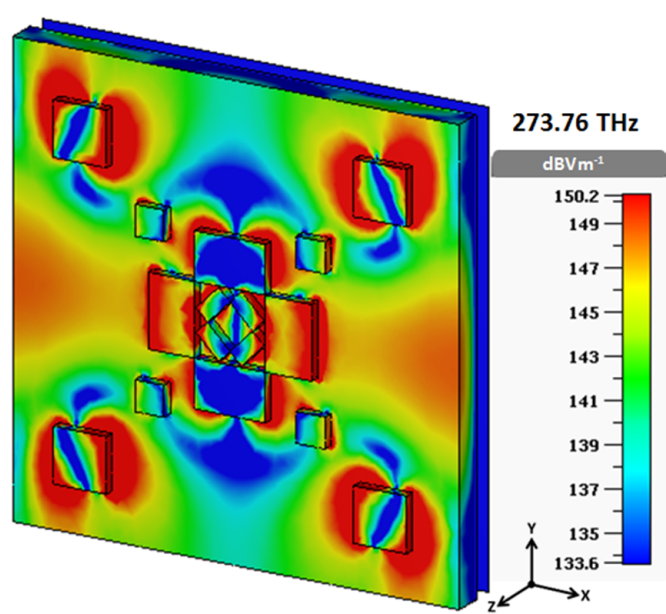

(a)

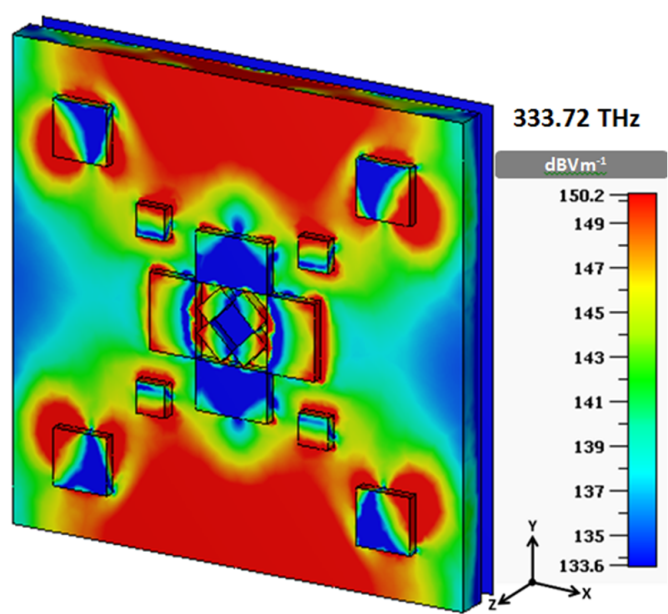

(b)

Figure 11. E-field distribution at (a) $273.76 \mathrm{THz}$ and (b) $333.72 \mathrm{THz}$.

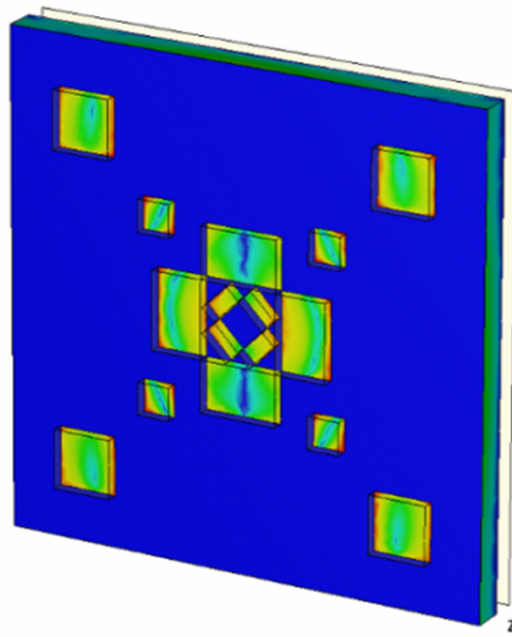

(a)

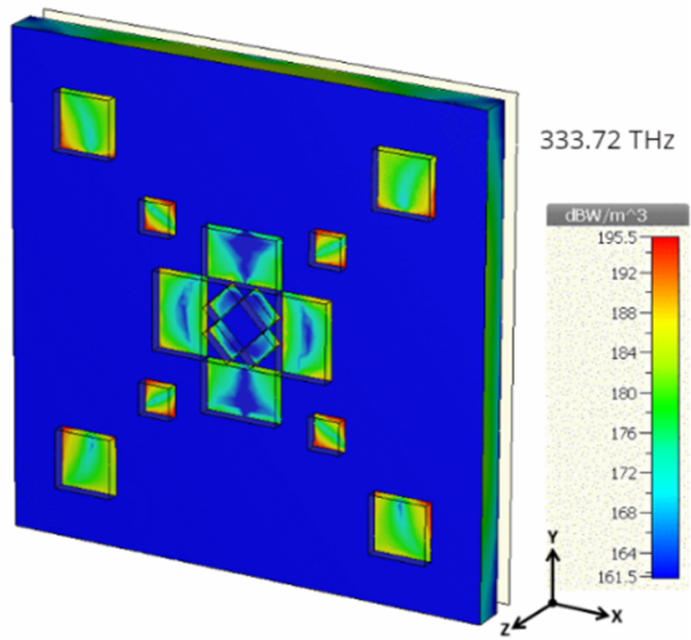

(b)

Figure 12. Power loss distribution at (a) $273.76 \mathrm{THz}$ and (b) $333.72 \mathrm{THz}$.

The proposed absorber is compared to absorbers from several recent studies comprising similar features; i.e., ultra-wideband absorption and operating at infrared frequency spectra. The comparison data in Table 2 show the proposed absorber has a significantly larger fractional and absolute bandwidth for long frequency range. The proposed absorber is constructed with comparatively low-cost materials, and offers polarization insensitivity while maintaining angular stability up to $35^{\circ}$. With all these characteristics, the proposed absorber is a considerable candidate for solar harvesting applications.

Table 2. Comparison with recent studies of ultra-wideband absorbers.

\begin{tabular}{|c|c|c|c|c|c|c|c|c|}
\hline Ref. & $\begin{array}{c}\text { Frequency } \\
\text { Range } \\
\text { THz }\end{array}$ & $\begin{array}{c}\text { Fractional } \\
\text { Bandwidth } \\
A(\omega)>90 \%\end{array}$ & $\begin{array}{c}\text { Absolute } \\
\text { Bandwidth } \\
\text { THz }\end{array}$ & $\begin{array}{l}\text { Material } \\
\text { Used }\end{array}$ & $\begin{array}{c}\text { Dimensionsz } \\
\quad \lambda(\mu \mathrm{m})\end{array}$ & $\begin{array}{l}\text { Polarization } \\
\text { insensitive }\end{array}$ & $\begin{array}{l}\text { Angular } \\
\text { Stability }\end{array}$ & Validation \\
\hline [43] & $136-374$ & $65.8 \%$ & 168 & $\mathrm{Au}, \mathrm{Ti}, \mathrm{SiO}_{2}$ & $0.27 \lambda \times 0.27 \lambda \times 0.13 \lambda$ & Yes & $\theta<40^{\circ}$ & Simulated \\
\hline [44] & 293-750 & $\begin{array}{c}72.2 \% \\
(\mathrm{~A}(\omega)<90 \%)\end{array}$ & $\begin{array}{c}331.42 \\
(\mathrm{~A}(\omega)<90 \%)\end{array}$ & $\begin{array}{c}\mathrm{Al}_{2} \mathrm{O}_{3 \prime} \\
\mathrm{Ge}_{2} \mathrm{Sb}_{2} \mathrm{Te}, \mathrm{Al}\end{array}$ & $0.4 \lambda \times 0.4 \lambda \times 0.21 \lambda$ & Yes & $\theta=0^{\circ}$ & Simulated \\
\hline [45] & $330-750$ & $56 \%$ & 291.46 & $\mathrm{Mn}, \mathrm{Al}_{2} \mathrm{O}_{3}$ & $0.6 \lambda \times 0.6 \lambda \times 0.3 \lambda$ & No & $\theta \leq 20^{\circ}$ & Measured \\
\hline [46] & $0.1-3.0$ & $187.09 \%$ & 2.9 & $\mathrm{Cu}, \mathrm{Si}$ & $5 \lambda \times 5 \lambda \times 0.15 \lambda$ & Yes & $\theta=0^{\circ}$ & Measured \\
\hline [47] & $21.43-37.47$ & $47.61 \%$ & 14.41 & $\mathrm{Ti}, \mathrm{Si}$ & $0.27 \lambda \times 0.27 \lambda \times 0.05 \lambda$ & No & $\theta=0^{\circ}$ & Simulated \\
\hline$[48]$ & $24.98-28.82$ & $3.7 \%$ & 1.07 & $\mathrm{In}_{2} \mathrm{SnO}_{5}, \mathrm{ZnS}$ & $0.86 \lambda \times 0.86 \lambda \times 0.055 \lambda$ & Yes & $\theta \leq 50^{\circ}$ & Measured \\
\hline This work & $220-360$ & $37.89 \%$ & 108 & $\mathrm{Cu}, \mathrm{GaAs}$ & $0.77 \lambda \times 0.77 \lambda \times 0.11 \lambda$ & Yes & $\theta \leq 35^{\circ}$ & Simulated \\
\hline
\end{tabular}




\section{Conclusions}

Metamaterial has been utilized to enhance the efficiency of solar energy harvesting. This study designed an ultra-broadband absorber in the infrared regime. The three-layered MA is designed with a copper resonator, where a GaAs substrate is sandwiched between the resonator and the ground layer. An equivalent circuit model is presented in order to validate the design and to understand resonant behavior. Although the solar spectrum is outstretched for a wide range of frequencies, the proposed absorber operates at the infrared regime, which covers more than $50 \%$ of the solar spectrum. By the proper tuning of geometric parameters, we obtained an optimized structure that provides near-unity absorption over an ultra-range of infrared frequency regime. The trends of absorption quality are also observed with the change in parameters. The proposed design offers absorption above $90 \%$ within a relative bandwidth of $37.89 \%$, which characterizes the design as an ultra-wideband absorber. The proposed unit cell also offers near-perfect absorption: $99.99 \%$ absorption for a vast portion of the frequency range. In addition, the absorber also offers angular stability for incident angles up to $35^{\circ}$, as well as exhibiting excellent insensitivity to polarization, due to the horizontal and rotational symmetry of the proposed absorber for TE-, TM- and TEM-polarized waves. All these features make the design a potential candidate for solar harvesting applications.

Author Contributions: Conceptualization, A.A., M.H.I., S.S.I., A.F.A. and M.T.I.; methodology, A.A., S.S.I., M.H.I., A.F.A. and M.T.I.; validation, S.S.I., A.A. and M.H.I.; formal analysis, A.A. and M.H.I.; investigation, A.A.; resources, S.S.I.; data curation, A.A. and S.S.I.; writing-original draft preparation, A.A.; writing-review and editing, S.S.I., A.F.A. and M.T.I.; visualization, A.A. and M.H.I.; supervision, S.S.I., A.F.A. and M.T.I.; project administration, M.T.I.; funding acquisition, A.F.A. and M.T.I.. All authors have read and agreed to the published version of the manuscript.

Funding: This research received no external funding.

Conflicts of Interest: The authors declare no conflict of interest.

\section{References}

1. Cui, T.J.; Smith, D.; Liu, R. (Eds.) Metamaterials; Springer: Boston, MA, USA, 2010; ISBN 978-1-4419-0572-7.

2. Ghafari, S.; Forouzeshfard, M.R.; Vafapour, Z. Thermo Optical Switching and Sensing Applications of an Infrared Metamaterial. IEEE Sens. J. 2020, 20, 3235-3241. [CrossRef]

3. Hoque, A.; Tariqul Islam, M.; Almutairi, A.; Alam, T.; Jit Singh, M.; Amin, N. A Polarization Independent Quasi-TEM Metamaterial Absorber for X and Ku Band Sensing Applications. Sensors 2018, 18, 4209. [CrossRef] [PubMed]

4. Lari, E.S.; Vafapour, Z.; Ghahraloud, H. Optically tunable triple-band perfect absorber for nonlinear optical liquids sensing. IEEE Sens. J. 2020. [CrossRef]

5. Hossain, M.J.; Karmaker, A.K.; Islam, M.J.; Ahmed, M.R.; Foysol, K.M. Polarization-Independent Perfect Metamaterial Absorber and Sensor Applications. DUET J. 2019, 5, 77-85.

6. Vafapour, Z. Polarization-Independent Perfect Optical Metamaterial Absorber as a Glucose Sensor in Food Industry Applications. IEEE Trans. Nanobiosci. 2019, 18, 622-627. [CrossRef] [PubMed]

7. Faruque, M.R.I.; Hossain, M.J.; Islam, S.S.; Jamlos, M.F.B.; Islam, M.T. Design and analysis of a new double C-shaped miniaturized metamaterial for multiband applications. Appl. Phys. A 2017, 123, 310. [CrossRef]

8. Islam, S.S.; Faruque, M.R.I.; Islam, M.T. A new double negative metamaterial for multi-band microwave applications. Appl. Phys. A 2014, 116, 723-733. [CrossRef]

9. Islam, S.S.; Tamim, A.M.; Faruque, M.R.I. A New Double-Negative Material for Multi-band Satellite Applications. In 10th International Conference on Robotics, Vision, Signal Processing and Power Applications; Zawawi, M.A.M., Teoh, S.S., Abdullah, N.B., Mohd Sazali, M.I.S., Eds.; Springer: Singapore, 2019; Volume 547, pp. 357-364. ISBN 9789811364464.

10. Islam, S.S.; Hasan, M.M.; Faruque, M.R.I. A new metamaterial-based wideband rectangular invisibility cloak. Appl. Phys. A 2018, 124, 160. [CrossRef]

11. Sunbeam Islam, S.; Rashed Iqbal Faruque, M.; Tariqul Islam, M. An ENG metamaterial based wideband electromagnetic cloak. Microw. Opt. Technol. Lett. 2016, 58, 2522-2525. [CrossRef] 
12. Guenneau, F.; Chakrabarti, S.; Guenneau, S.; Ramakrishna, S.A. Origami with negative refractive index to generate super-lenses. J. Phys. Condens. Matter 2014, 26, 405303. [CrossRef]

13. Marques, R.; Mesa, F.; Freire, M.J.; Baena, J.D. Image formation and detection in metamaterial super-lenses. In Proceedings of the 2005 18th International Conference on Applied Electromagnetics and Communications, Dubrovnik, Croatia, 12-14 October 2005; pp. 1-4.

14. Salleh, A.S.; Yang, C.C.; Alam, T.; Singh, M.; Singh, J.; Samsuzzaman, M.; Islam, M.T. Development of Microwave Brain Stroke Imaging System using Multiple Antipodal Vivaldi Antennas Based on Raspberry Pi Technology. J. Kejuruter. (J. Eng.) 2020, 32, 39-49.

15. Landy, N.I.; Sajuyigbe, S.; Mock, J.J.; Smith, D.R.; Padilla, W.J. A Perfect Metamaterial Absorber. Phys. Rev. Lett. 2008, 100, 207402. [CrossRef] [PubMed]

16. Tonouchi, M. Cutting-edge terahertz technology. Nat. Photonics 2007, 1, 97-105. [CrossRef]

17. Williams, G.P. Filling the THz gap—high power sources and applications. Rep. Prog. Phys. 2005, 69, 301-326. [CrossRef]

18. Rufangura, P.; Sabah, C. Dual-band perfect metamaterial absorber for solar cell applications. Vacuum 2015, 120, 68-74. [CrossRef]

19. Bağmanc1, M.; Karaaslan, M.; Ünal, E.; Akgol, O.; Sabah, C. Extremely-broad band metamaterial absorber for solar energy harvesting based on star shaped resonator. Opt. Quantum Electron. 2017, 49, 257. [CrossRef]

20. Azad, A.K.; Kort-Kamp, W.J.M.; Sykora, M.; Weisse-Bernstein, N.R.; Luk, T.S.; Taylor, A.J.; Dalvit, D.A.R.; Chen, H.-T. Metasurface Broadband Solar Absorber. Sci. Rep. 2016, 6. [CrossRef]

21. Karaaslan, M.; Unal, E.; Akgol, O.; Bağmanc1, M.; Bakir, M.; Sabah, C. Solar energy harvesting with ultra-broadband metamaterial absorber. Int. J. Mod. Phys. B 2019, 33. [CrossRef]

22. Landy, N.I.; Bingham, C.M.; Tyler, T.; Jokerst, N.; Smith, D.R.; Padilla, W.J. Design, theory, and measurement of a polarization insensitive absorber for terahertz imaging. Phys. Rev. B 2009, 79, 125104. [CrossRef]

23. Dincer, F.; Akgol, O.; Karaaslan, M.; Unal, E.; Sabah, C. Polarization Angle Independent Perfect Metamaterial Absorbers for Solar Cell Applications in the Microwave, Infrared, and Visible Regime. Prog. Electromagn. Res. 2014, 144, 93-101. [CrossRef]

24. Kim, Y.J.; Kim, J.M.; Yoo, Y.J.; Van Tuong, P.; Zheng, H.; Rhee, J.Y.; Lee, Y. Dual-absorption metamaterial controlled by electromagnetic polarization. J. Opt. Soc. Am. B 2014, 31, 2744-2747. [CrossRef]

25. Gomes de Souza, I.L.; Rodriguez-Esquerre, V.F. Omnidirectional broadband absorber for visible light based on a modulated plasmonic multistack grating. Opt. Laser Technol. 2020, 124, 105981. [CrossRef]

26. Dhillon, A.S.; Mittal, D. Wide band ultrathin polarization insensitive electric field driven metamaterial absorber. Opt. Commun. 2019, 443, 186-196. [CrossRef]

27. Liu, Z.; Zhang, H.; Fu, G.; Liu, G.; Liu, X.; Yuan, W.; Xie, Z.; Tang, C. Colloid templated semiconductor meta-surface for ultra-broadband solar energy absorber. Sol. Energy 2020, 198, 194-201. [CrossRef]

28. Lee, D.-H.; Ling, K.; Lim, S.; Baek, C.-W. Fabrication of polarization-insensitive, multi-resonant metamaterial absorber using wafer bonding of glass dielectric substrate. Microelectron. Eng. 2015, 136, 42-47. [CrossRef]

29. Tao, H.; Landy, N.I.; Bingham, C.M.; Zhang, X.; Averitt, R.D.; Padilla, W.J. A metamaterial absorber for the terahertz regime: Design, fabrication and characterization. Opt. Express 2008, 16, 7181. [CrossRef]

30. Wang, W.; Chen, Y.; Yang, S.; Zheng, X.; Cao, Q. Design of a broadband electromagnetic wave absorber using a metamaterial technology. J. Electromagn. Waves Appl. 2015, 29, 2080-2091. [CrossRef]

31. Huang, T.-Y.; Tseng, C.-W.; Yeh, T.-T.; Yeh, T.-T.; Luo, C.-W.; Akalin, T. Experimental realization of ultrathin, double-sided metamaterial perfect absorber at terahertz gap through stochastic design process. Sci. Rep. 2015, 5, 18605. [CrossRef]

32. Wen, Y.; Ma, W.; Bailey, J.; Matmon, G.; Yu, X. Broadband Terahertz Metamaterial Absorber Based on Asymmetric Resonators With Perfect Absorption. IEEE Trans. Terahertz Sci. Technol. 2015, 5, 406-411. [CrossRef]

33. Grant, J.; Ma, Y.; Saha, S.; Khalid, A.; Cumming, D.R.S. Polarization insensitive, broadband terahertz metamaterial absorber. Opt. Lett. 2011, 36, 3476. [CrossRef]

34. Chen, X.; Fan, W. Ultra-flexible polarization-insensitive multiband terahertz metamaterial absorber. Appl. Opt. 2015, 54, 2376. [CrossRef] [PubMed]

35. Soheilifar, M.R.; Sadeghzadeh, R.A. Design, fabrication and characterization of stacked layers planar broadband metamaterial absorber at microwave frequency. AEU-Int. J. Electron. Commun. 2015, 69, $126-132$. [CrossRef] 
36. Li, W.; Xiao, Z.; Ling, X.; Zheng, X.; Li, C.; Zou, H. Design of a broadband, wide-angle metamaterial absorber base on hybrid structure. J. Electromagn. Waves Appl. 2018, 32, 651-660. [CrossRef]

37. Liu, H.; Wang, Z.-H.; Li, L.; Fan, Y.-X.; Tao, Z.-Y. Vanadium dioxide-assisted broadband tunable terahertz metamaterial absorber. Sci. Rep. 2019, 9, 5751. [CrossRef] [PubMed]

38. Paul, C.R. Inductance: Loop and Partial; Wiley: Piscataway, NJ, USA; IEEE: Hoboken, NJ, USA, 2010; ISBN 978-0-470-46188-4.

39. Grover, F.W. Inductance Calculation, 1st ed.; Dover Publication Inc.: New York, NY, USA, 1946.

40. Pendry, J.B.; Holden, A.J.; Robbins, D.J.; Stewart, W.J. Magnetism from conductors and enhanced nonlinear phenomena. IEEE Trans. Microw. Theory Technol. 1999, 47, 2075-2084. [CrossRef]

41. Watts, C.M.; Liu, X.; Padilla, W.J. Metamaterial Electromagnetic Wave Absorbers. Adv. Mater. 2012, 24, OP98-OP120. [CrossRef] [PubMed]

42. Murata, K.; Matsumoto, J.; Tezuka, A.; Matsuba, Y.; Yokoyama, H. Super-fine ink-jet printing: Toward the minimal manufacturing system. Microsyst. Technol. 2005, 12, 2. [CrossRef]

43. Ding, F.; Dai, J.; Chen, Y.; Zhu, J.; Jin, Y.; Bozhevolnyi, S.I. Broadband near-infrared metamaterial absorbers utilizing highly lossy metals. Sci. Rep. 2016, 6, 39445. [CrossRef]

44. Mou, N.; Liu, X.; Wei, T.; Dong, H.; He, Q.; Zhou, L.; Zhang, Y.; Zhang, L.; Sun, S. Large-scale, low-cost, broadband and tunable perfect optical absorber based on phase-change material. Nanoscale 2020, 12, 5374-5379. [CrossRef]

45. Aalizadeh, M.; Khavasi, A.; Butun, B.; Ozbay, E. Large-Area, Cost-Effective, Ultra-Broadband Perfect Absorber Utilizing Manganese in Metal-Insulator-Metal Structure. Sci. Rep. 2018, 8, 9162. [CrossRef]

46. Xiao, D.; Zhu, M.; Sun, L.; Zhao, C.; Wang, Y.; Tong Teo, E.H.; Hu, F.; Tu, L. Flexible Ultra-Wideband Terahertz Absorber Based on Vertically Aligned Carbon Nanotubes. ACS Appl. Mater. Interfaces 2019, 11, 43671-43680. [CrossRef] [PubMed]

47. Qin, Z.; Meng, D.; Xiong, Y.; Tang, Y.; Zhou, Y.; Shi, X.; Zhang, Y.; Hou, E.; Liang, Z. Small-period Ultra-broadband Long-wavelength Infrared Metamaterial Absorber. In Proceedings of the 2019 Photonics \& Electromagnetics Research Symposium-Fall (PIERS-Fall), Xiamen, China, 17-20 December 2019; pp. 3322-3328.

48. Yang, J.; Xu, C.; Qu, S.; Ma, H.; Wang, J.; Pang, Y. Optical transparent infrared high absorption metamaterial absorbers. J. Adv. Dielectr. 2018, 8, 1850007. [CrossRef] 